九州大学学術情報リポジトリ

Kyushu University Institutional Repository

\title{
Richards Function in Animal Growth Analysis and Friedmann Equation in Space Expansion Analysis
}

Shimojo, Masataka

Laboratory of Regulation in Metabolism and Behavior, Division of Animal and Marine Bioresource

Sciences, Department of Bioresource Sciences, Faculty of Agriculture, Kyushu University

https://doi.org/10.5109/1800843

出版情報：九州大学大学院農学研究院紀要. 62 (1)，pp.105-109，2017-02-24. Faculty of Agriculture, Kyushu University

バージョン :

権利関係 : 


\title{
Richards Function in Animal Growth Analysis and Friedmann Equation in Space Expansion Analysis
}

\author{
Masataka SHIMOJO* \\ Laboratory of Regulation in Metabolism and Behavior, Division of Animal and Marine Bioresource Sciences, \\ Department of Bioresource Sciences, Faculty of Agriculture, Kyushu University, \\ Fukuoka 812-8581, Japan \\ (Received November 4, 2016 and accepted November 4, 2016)
}

\begin{abstract}
This study was designed to investigate the analytical concept common to animal growth analysis and space expansion analysis. In addition, the space expansion was investigated using the equivalence principle. Three differential equations for animal growth analysis were obtained from Richards function. Three differential equations for space expansion analysis were Friedmann equations and deceleration parameter. The results obtained were as follows. (I) Three differential equations in both analyses were characterized as follows; (a) the square of the relative growth (or expansion) rate, (b) the relative growth (or expansion) acceleration rate, (c) the equation given by (b)/(a). The analytical concept of differential equation was the same between animal growth analysis and space expansion analysis. This commonness might be ascribed to the mathematical characteristics of the differentiation of exponential function, though the analytical complexity was different between animal growth analysis and space expansion analysis. (II) The increase in the cosmic scale factor in the flat FLRW space-time was related to the decrease in the recession velocity of the illuminant in the Minkowski space-time, an accumulation of equivalence principle suggesting a relationship between the cosmic scale factor and the Lorentz factor. The real expansion of the flat FLRW space-time might be interpreted using the Bondi $\mathrm{K}$-factor elongation of the Minkowski space-time that did not contain the gravity.
\end{abstract}

Key words: animal growth, common structure, Friedmann equation, Richards function, space expansion

\section{INTRODUCTION}

Tegmark (2014) suggests that the growth of a human baby resembles the expansion of our baby universe based on the inflationary theory. In previous reports (Shimojo, 2016a, 2016b) on the mathematical structure common to animal growth analysis and space expansion analysis, the analysis of animal growth was mainly based on Bertalanffy function (Bertalanffy, 1957). Bertalanffy function, however, has a complex structure that is difficult to manipulate.

In the present study, Richards function (Richards, 1959), an abbreviation of Bertalanffy function, was used to investigate the analytical concept common to animal growth analysis and space expansion analysis. In addition, the space expansion was investigated using the equivalence principle.

\section{ANIMAL GROWTH ANALYSIS AND SPACE EXPANSION ANALYSIS}

\section{Richards function in animal growth analysis}

Richards (1959), by abbreviating Bertalanffy function (1) (Bertalanffy, 1957), gave Richards function (2),

$$
\begin{aligned}
W & =\left(\eta / \kappa-\left(\eta / \kappa-W_{0}^{1-m}\right) \exp (-\kappa(1-m) t)\right)^{1 /(1-m)} \\
& =(\eta / \kappa)^{1 /(1-m)}\left(1-\kappa / \eta\left(\eta / \kappa-W_{0}^{1-m}\right) \exp (-\kappa(1-m) t)\right)^{1 /(1-m)}
\end{aligned}
$$

\footnotetext{
* E-mail: mshimojo@agr.kyushu-u.ac.jp

This study was conducted mostly at Agri-Bio Research Laboratory on Ito Campus, Kyushu University, Motooka, Fukuoka 819-0395
}

$$
=A\left(1-b \cdot \exp (-k \cdot t)^{1 /(1-m)}\right.
$$

where $W=$ weight, $\eta=$ anabolic constant, $\kappa=$ catabolic constant, $t=$ time, $m=$ constant, $W_{0}^{1-m}=$ weight at $t=0$, $A, b, k$ are constants.

Three differential equations for animal growth analysis [(3) (5)] are obtained from (2),

$$
\begin{aligned}
& \left(\frac{1}{W} \cdot \frac{d W}{d t}\right)^{2}=\left(\frac{b \cdot k \cdot \exp (-k \cdot t)}{(1-m)(1-b \cdot \exp (-k \cdot t))}\right)^{2}, \\
& \frac{1}{W} \cdot \frac{d^{2} W}{d t^{2}}=\frac{(b \cdot \exp (-k \cdot t)+m-1) b \cdot k^{2} \cdot \exp (-k \cdot t)}{(1-m)^{2}(1-b \cdot \exp (-k \cdot t))^{2}}
\end{aligned}
$$

$$
\begin{aligned}
& \frac{\text { Eq. }(4)}{\text { Eq.(3) }}=\frac{(1 / W)\left(d^{2} W / d t^{2}\right)}{((1 / W)(d W / d t))^{2}} \\
& =\frac{W\left(d^{2} W / d t^{2}\right)}{(d W / d t)^{2}}=\frac{b \cdot \exp (-k \cdot t)+m-1}{b \cdot \exp (-k \cdot t)} .
\end{aligned}
$$

Shimojo et al. (2012) suggest that differential equation (5) gives a unified viewpoint, which classifies five functions into three groups; group-1 (Richards function), group-2 (Mitscherlich function, logistic function, Gompertz function), and group-3 (basic growth function). Basic growth function does not have an asymptote in contrary to the other four functions. 


\section{Friedmann equation in space expansion analysis}

Friedmann-Lemaitre-Robertson-Walker (FLRW) metric is given by (6),

$$
d s^{2}=-c^{2} d t^{2}+\alpha^{2}(t)\left[\frac{d r^{2}}{1-K r^{2}}+r^{2}\left(d \theta^{2}+\sin ^{2} \theta d \phi^{2}\right)\right],
$$

where $s=$ space-time interval, $c=$ speed of light in vacuum, $t=$ time, $a=$ cosmic scale factor, $K=$ curvature of space, $(r, \theta, \phi)=$ spherical polar coordinates.

Three differential equations for space expansion analysis using the cosmic scale factor in (6) are Friedmann equations [(7), (8)] and deceleration parameter (9),

$$
\begin{aligned}
& \left(\frac{1}{a} \cdot \frac{d a}{d t}\right)^{2}=\frac{8 \pi G}{3 c^{2}} \rho-\frac{c^{2} K}{a^{2}}+\frac{c^{2} \Lambda}{3}, \\
& \frac{1}{a} \cdot \frac{d^{2} a}{d t^{2}}=-\frac{4 \pi G}{3 c^{2}}(\rho+3 p)+\frac{c^{2} \Lambda}{3}, \\
& \frac{\text { Eq.(8) }}{\text { Eq.(7) }}=\frac{(1 / a)\left(d^{2} a / d t^{2}\right)}{((1 / a)(d a / d t))^{2}} \\
& =\frac{a\left(d^{2} a / d t^{2}\right)}{(d a / d t)^{2}}=-q,
\end{aligned}
$$

where $\pi=$ circular constant, $G=$ gravitational constant, $\rho=$ energy density, $\Lambda=$ cosmological constant, $p=$ pressure, $q=$ deceleration parameter.

\section{Analytical concept common to animal growth anal- ysis and space expansion analysis}

Equation (3) shows the square of the relative growth rate of the animal, and equation (7) shows the square of the relative expansion rate of the space. Equation (4) shows the relative growth acceleration rate of the animal, and equation (8) shows the relative expansion acceleration rate of the space. Equation (5) is obtained by (4)/ (3), and equation (9) is obtained by (8)/(7).

Comparing animal growth analysis [(3), (4), (5)] and space expansion analysis [(7), (8), (9)] suggests (A) (C).

(A) Since the mathematical structure of the lefthand side of differential equation is the same between (3) and (7), between (4) and (8), and between (5) and (9), the analytical concept is the same between animal growth analysis and space expansion analysis.

(B) Since the mathematical structure of the righthand side of differential equation is different, the analytical complexity is different between animal growth analysis and space expansion analysis.

(C) Richards function (2) shows a flexibility in the description of various animal growth curves because parameters change the position of inflection point, and in the special case there is an exponential growth. Friedmann equations [(7), (8)] show a flexibility in the description of various space expansion curves because parameters change the position of inflection point, and in the special case there is an exponential expansion.

The above commonness might be ascribed to the mathematical characteristics of the differentiation of exponential function, though the analytical complexity is different between animal growth analysis and space expansion analysis.

\section{SPACE EXPANSION ANALYSIS}

\section{FLRW metric and Minkowski metric}

$$
d s^{2}=-c^{2} d t^{2}+a^{2}(t)\left[\frac{d r^{2}}{1-K r^{2}}+r^{2}\left(d \theta^{2}+\sin ^{2} \theta d \phi^{2}\right)\right] .
$$

The FLRW metric (6) suggests (I) (III). (I) The initial size of the FLRW space-time is given by $a(0)=0$. (II) The FLRW space-time that is expanding is nearly flat $(K$ $\approx 0)$. Since the critical density is very low $\left(\approx 10^{-29} h^{2} \mathrm{~g} /\right.$ $\mathrm{cm}^{3}$ ), there are lots of voids in the flat FLRW spacetime. (III) The present size of the FLRW space-time is given by $a\left(t_{\mathbf{0}}\right)=1$, the normalization that is generally used.

When $a=1$ and $K=0$, the FLRW metric (6) leads to the Minkowski metric (10),

$$
d s^{2}=-c^{2} d t^{2}+d r^{2}+r^{2}\left(d \theta^{2}+\sin ^{2} \theta d \phi^{2}\right) .
$$

The FLRW space-time is nearly flat $(K \approx 0)$ due to the presence of the gravity, but the Minkowski spacetime is flat $(K=0)$ due to the absence of the gravity.

\section{Increase in wavelength, space expansion, and moving illuminant}

What really occurs in the flat FLRW space-time is the space expansion that causes the increase in wavelength (cosmolocial redshift), where the space between illuminants is expanding.

$$
\frac{\lambda_{o}}{\lambda_{E}}=\frac{a\left(t_{o}\right)}{a\left(t_{E}\right)}=\frac{1}{a\left(t_{E}\right)},
$$

where $\lambda_{E}=$ emitted wavelength, $\lambda_{\mathbf{o}}=$ observed wavelength, $a\left(t_{E}\right)=$ size of the cosmic scale factor when light was emitted, $a\left(t_{\mathbf{0}}\right)=$ size of the cosmic scale factor when light was observed, the present size of the space is given by normalizing the cosmic scale factor $\left[\alpha\left(t_{\mathbf{o}}\right)=1\right]$.

Tegmark (2014) shows that the general relativity allows the two different viewpoints to be equally valid in the flat FLRW space-time; space is expanding but illuminants are not moving, space is not expanding but illuminants are moving.

By the way, if the same increase in wavelength occurs in the Minkowski space-time, then this is caused by the recession of the illuminant and is called the relativistic Doppler redshift (Einstein, 1905). 


$$
\frac{\lambda_{O}}{\lambda_{E}}=\sqrt{\frac{1+\beta_{E}}{1-\beta_{E}}}=\frac{1}{\sqrt{\frac{1-\beta_{E}}{1+\beta_{E}}}},
$$

where $1 \geq \beta_{\boldsymbol{E}} \geq 0, \beta_{\boldsymbol{E}}=v_{\boldsymbol{E}} / \mathrm{c}, v_{\boldsymbol{E}}=$ recession velocity of the illuminant when light was emitted.

Expression (11) is based on the general relativity, but expression (12) is based on the special relativity. Despite this difference, mathematically relating expressions (11) and (12) suggests expressions (13) (16),

$$
\begin{aligned}
& a(t)=\sqrt{\frac{1-\beta}{1+\beta}}, \\
& \frac{1-a^{2}(t)}{1+a^{2}(t)}=\beta, \\
& \frac{1+a^{2}(t)}{2 a(t)}=\frac{1}{\sqrt{1-\beta^{2}}}, \\
& \frac{1-a^{2}(t)}{2 a(t)}=\frac{\beta}{\sqrt{1-\beta^{2}}},
\end{aligned}
$$

where $0 \leq a(t) \leq 1,1 \geq \beta \geq 0, \beta=v / c$.

Due to the same condition of three kinds [the same increase in wavelength, the same size of the initial space $(=0)$, the same size of the present space $(=1)]$, expression (13) suggests that the increase in the cosmic scale factor in the flat FLRW space-time is related to the decrease in the recession velocity of the illuminant in the Minkowski space-time. This slightly resembles the Hubble's law (1929). Expressions (14) (16) suggest a relationship between the cosmic scale factor and the Lorentz factor. Those phenomena suggest an accumulation of equivalence principle that occurs along the space expansion. In fact, however, the illuminant is present on the expansion curve that the cosmic scale factor describes in the flat FLRW space-time. The equivalence principle suggests that the illuminant at time $t_{\boldsymbol{E}}$ in the flat FLRW space-time is interpreted as the illuminant having the recession velocity $v_{\boldsymbol{E}}\left(v_{\boldsymbol{E}} / c=\beta_{\boldsymbol{E}}\right)$ in the Minkowski space-time.

\section{Real expansion of flat FLRW space-time and Bondi elongation of Minkowski space-time}

The flat FLRW space-time shows the real expansion as a result of the balance between the attractive gravity and the repulsive gravity, but the Minkowski space-time shows the Bondi K-factor (Bondi, 1964) elongation under the absence of the gravity. The real expansion applies to everywhere in the flat FLRW space-time, and the Bondi elongation applies to everywhere in the Minkowski spacetime. Those difference and similarity suggest (a) (c).

(a) The real expansion of the flat FLRW space-time might be interpreted using the Bondi elongation of the Minkowski space-time that does not contain the gravity. (b) Introducing the gravity into the Bondi elongation of the Minkowski space-time might lead to the real expansion of the flat FLRW space-time. (c) The phenomena (a) and (b) might be due somewhat to the very low critical density $\left(\approx 10^{-29} h^{2} \mathrm{~g} / \mathrm{cm}^{3}\right)$ of the flat FLRW space-time.

By the way, Maldacena (2005) proposes that the gravity theory in the 5-dimensional anti-de Sitter spaces is described using the 4-dimensional gauge theory in the conformal field theories that do not contain the gravity.

\section{Scale invariant and choice of coordinate system}

$$
a(t)=\sqrt{\frac{1-\beta}{1+\beta}} .
$$

The right-hand side of (13) suggests that the shape of the elongation curve of toy space model is similar even when the length of time from the birth is different, because $\beta(=v / c)$ is independent of the difference in the length of time. If the size of the cosmic scale factor at the arbitrary time when the light is observed is normalized to 1 , then expression (13) suggests that the shape of the expansion curve that the cosmic scale factor describes is similar even when the length of time from the birth is different.

In addition, expression (13) suggests that the shape of the space expansion curve is not dependent on the choice of the coordinate system between the flat FLRW space-time and the Minkowski space-time, in other words, is not dependent on the presence or absence of the gravity. This suggests that the general relativity and the special relativity take the same shape in the relativistic expansion curve of space-time.

The above two phenomena, however, are contradictory to the coincidence problem and cosmological constant problem in the $\Lambda \mathrm{CDM}$ model. The correspondence between the cosmic scale factor and this toy space model in (13) requires one-to-one correspondence between time $t$ of $a(t)$ in the FLRW space-time and the recession velocity $v(v / c=\beta)$ of the illuminant in the Minkowski space-time.

\section{Cosmological redshift and relativistic Doppler redshift}

Hubble (1929), using the relativistic Doppler redshift, showed that the recession velocity of the illuminant was proportional to the distance to the illuminant. This Hubble's law gave an evidence of the cosmic expansion causing the cosmological redshift. The increase in wavelength in the flat FLRW space-time depends on the increase of the cosmic scale factor in the whole period from the emission to the absorption of light (Weinberg, 2008).

In the whole period from the emission to the absorption of light, expression (13) suggests a correspondence between the increase of the cosmic scale factor in the flat FLRW space-time and the decrease of the recession velocity of the illuminant in the Minkowski space-time. This slightly resembles the Hubble's law (1929). Many preceding studies had already noticed this correspondence, as shown in $(d) \sim(f)$. 
(d) The accumulation of many infinitesimal relativistic Doppler redshifts leads to the cosmological redshift (Bunn and Hogg, 2009; Matsubara, 2010). (e) The redshift, defined as the effect of the space expansion, is related also to the recession velocity of the illuminant (Tsujikawa, 2013; Pierseaux, 2014; Kohri, 2016). (f) There is a possible use of the relativistic Doppler redshift as an alternative to the cosmological redshift (Macleod, 2004; Tanighchi, 2005; Chodorowski, 2006; Sitnikov, 2006; Davis, 2010; Farley, 2010; Mei and Yu, 2013; Li, 2014; Tatum et al., 2015).

\section{Bondi elongation of toy space model and its appli- cation to the real expansion of the space}

As suggested in reports (Shimojo, 2016a, 2016b), this toy space model in the Minkowski space-time is composed of space size (17), energy density (18) and conservation of energy (19),

$$
\begin{aligned}
& M(\beta)=\sqrt{\frac{1-\beta}{1+\beta}}, \text { (17) } E(\beta)=\sqrt{\frac{1+\beta}{1-\beta}}, \\
& M(\beta) \cdot E(\beta)=\sqrt{\frac{1-\beta}{1+\beta}} \sqrt{\frac{1+\beta}{1-\beta}}=1,
\end{aligned}
$$

where $1 \geq \beta \geq 0,0 \leq M(\beta) \leq 1, \infty>E(\beta) \geq 1$.

If $\beta=1$ in (19), then (20) is given. The natural logarithm of (20) suggests (21) (29).

$$
\begin{aligned}
& M(1) \cdot E(1)=0 \cdot \infty=1 . \\
& \ln (0)+\ln (\infty)=\ln (1) .
\end{aligned}
$$

Thus,

$$
\begin{aligned}
& -\infty+\infty \\
& =2 n \pi \boldsymbol{i},
\end{aligned}
$$

$$
=2 n \pi \cdot \lim _{v \rightarrow \infty} \sqrt{\frac{c / v+1}{c / v-1}},
$$

$$
=2 n \pi \cdot \lim _{v \rightarrow \infty} \sqrt{\frac{c+v}{c-v}},
$$

$$
\begin{aligned}
& =2 n \pi \cdot \lim _{\Delta \boldsymbol{t} \rightarrow \mathbf{0}} \sqrt{\frac{c+(\Delta x / \Delta t)}{c-(\Delta x / \Delta t)}},(\text { where } \Delta x>0), \\
& \geqq 2 n \pi \cdot \frac{[\hat{q}, \hat{p}]}{2 \Delta E \cdot \Delta t}, \\
& =2 n \pi \cdot\left(c \alpha \cdot \hat{p}+\beta m c^{2}\right) \psi /\left(\frac{h}{2 \pi} \cdot \frac{\partial \psi}{\partial t}\right), \\
& =2 n \pi \cdot \sqrt{\frac{\Lambda g_{u v}}{G_{u v}-\left(8 \pi G / c^{4}\right) T_{u v}}},
\end{aligned}
$$

$$
=0 \text {, (when } n=0) \text {. }
$$

The Bondi K-factor elongation of toy space model is taken up again [(i) (vi)], by adding some explanations to previous reports (Shimojo, 2014, 2015a, 2015b, 2016a, 2016b).

(i) There is a singularity (20) that is related to the birth of toy space model. This slightly resembles the singularity that Hawking and Penrose (1970) showed in the general relativity. A pseudo-renormalization of the infinity gives a multivalued function (22), suggesting that the singularity of toy space model (20) is related to the quantum theory [(26), (27)] and general relativity theory (28). This singularity might be unstable due to the quantum uncertainty (26). The imaginary unit in the quantum theory might be associated with the instantaneous action at a distance (25) that Einstein did not approve due to the Lorentz violation [(23), (24)]. Why we think the quantum phenomena mysterious might be due to the mathematical phenomenon that relates the imaginary unit to the infinite velocity [(23) (25)]. This mathematical phenomenon, though looks absurd due to the Lorentz invariant breaking, might play some role in the wave-particle duality, electron cloud, quantum superposition, wave function collapse, quantum entanglement and so on. John Bell tells that there is a deeper level that is not Lorentz invariant behind the apparent Lorentz invariant (Davies and Brown, 1986). From the seeming zero (29), there might be an emergence of quantum phenomena [(26), (27)], infinitesimal distance and infinitesimal time (25) and general relativity (28).

(ii) The extremely high density of energy $(\approx \infty)$ causes an early rapid Bondi elongation of toy space model, where the time dilation caused by $\beta \approx 1$ might give enough time to keep the early elongation rate extremely high. This slightly resembles the slow-roll inflation of the standard cosmic model proposed by theoretical studies (Linde, 1982; Albrecht and Steinhardt, 1982).

(iii) After the early rapid elongation, this toy space model shows a decelerated elongation. This slightly resembles the standard cosmic model that shows the decelerated expansion after the early inflation. Contrary to the standard cosmic model, this toy space model does not show the cosmic recombination.

(iv) This toy space model has an inflection point at which the decelerated elongation is switched to the accelerated elongation that continues until now. This slightly resembles the observational results based on the standard cosmic model (Riess et al., 1998; Perlmutter et al., 1999).

(v) The conservation of energy (19) in toy space model suggests that the energy density is much lower at the present time $(=1)$ than at the birth time $(\approx \infty)$. This slightly resembles the observational result based on the standard cosmic model showing that the present energy density is $1 / 10^{117}$ of the inflationary vacuum energy density (Matsubara, 2010). If the size of toy space model becomes $\infty$, then the energy density becomes 0 . This is, however, contradictory to the standard cosmic model that shows an increase in the amount of dark energy 


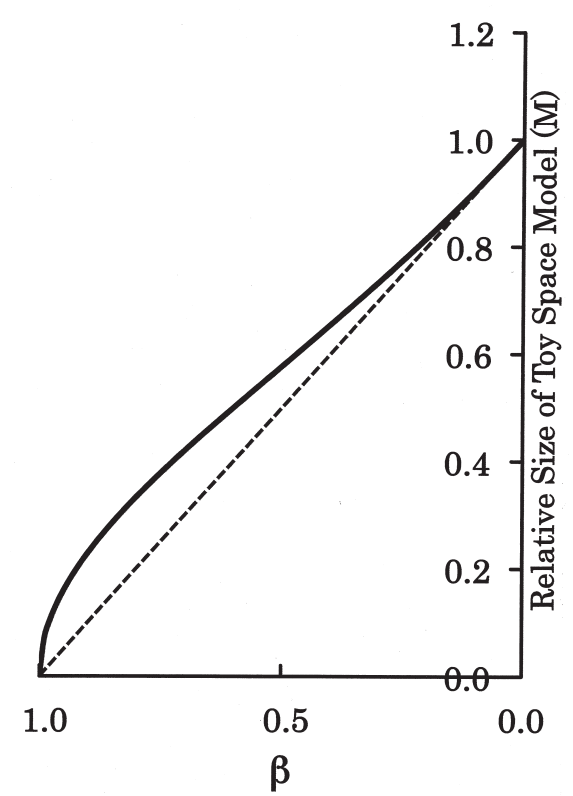

Fig. 1. Bondi K-factor elongation of toy space model $(M)$ under the domain of $1 \geq \beta \geq$ 0 , where $\beta=v / c$. The solid line shows the Bondi elongation curve, and the broken line shows the straight elongation without deceleration or acceleration.

along with the cosmic expansion.

(vi) Fig. 1 shows that the difference between Bondi elongation and straight elongation suggests the effect of special relativity on space-time.

Despite the above differences, generalizing the Bondi elongation of toy space model that does not contain the gravity to the expansion of the standard cosmic model might lead to the emergence of dark matter and dark energy.

\section{REFERENCES}

Albrecht, A. and P. J. Steinhardt 1982 Cosmology for grand unified theories with radiatively induced symmetry breaking. Phys. Rev. Lett., 48: 1220-1223

Bertalanffy, L. von. 1957 Quantitative laws in metabolism and growth. Quart. Rev. Biol., 32: 217-231

Bondi, H. 1964 Relativity and Common Sense (Doubleday and Company Inc., USA). Japanese Translation by T. Yamanouchi for publication (1967), Kawade Shobo Publishers, Tokyo.

Bunn E. F. and D. W. Hogg 2009 The kinematic origin of the cosmological redshift. arXiv:0808.1081v2

Chodorowski, M. J. 2006 Is space really expanding? A counterexample. arXiv.astro-ph/0601171v2

Davies, P. C. W. and J. R. Brown 1986 The Ghost in the Atom. Cambridge University Press, Cambridge.

Davis, T. M. 2010 Is the universe leaking energy? Sci. Am., 303: 32-39

Einstein, A. 1905 Zur Elektrodynamik bewegter Körper. Ann. Phys. (Leipzig), 322: 891-921

Farley, F. J. M. 2010 Does gravity operate between galaxies? Observational evidence re-examined. Proc. R. Soc. A, 466: 3089-3906

Hawking, S. W. and R. Penrose 1970 The singularities of gravitational collapse and cosmology. Proc. Roy. Soc. London, A314: $529-548$

Hubble, E. 1929 A relation between distance and radial velocity among extra-galactic nebulae. Proc. Natl. Acad. Sci. USA, 15: $168-173$

Kohri, K. 2016 What kind of space-time is the universe made of? Beret Publishing Co., Ltd., Tokyo (written in Japanese)

Li, M. 2014 Understanding the gravitational and cosmological redshifts as Doppler shifts by gravitational phase factors. arXiv: $1401.5337 \mathrm{v} 2$

Linde, A. D. 1982 A new inflationary universe scenario: a possible solution of the horizon, flatness, homogeneity, isotropy and primordial monopole problems. Phys. Lett., 108B: 389-393

Macleod, A. 2004 Redshift and energy conservation. arXiv.org/ pdf/physics/0407077

Maldacena, J. 2005 The illusion of gravity. Sci. Am., 293: 56-63

Matsubara, T. 2010 Modern Cosmology. University of Tokyo Press, Tokyo (written in Japanese)

Mei, X. and P. Yu 2013 Cosmology should directly use the Doppler's formula to calculate the red shift of Ia supernova Int. J. Astron. Astrophys., 3: 303-317

Perlmutter, S., G. Aldering, G. Goldhaber, R. A. Knop, P. Nugent, P. G. Castro, S. Deustua, S. Fabbro, A. Goobar, D. E. Groom, I. M. Hook, A. G. Kim, M. Y. Kim, J. C. Lee, N. J. Nunes, R. Pain, C. R. Pennypacker, R. Quimby, C. Lidman, R. S. Ellis, M. Irwin, R. G. McMahon, P. Ruiz-Lapuente, N. Walton, B. Schaefer, B. J. Boyle, A. V. Filippenko, T. Matheson, A. S. Fruchter, N. Panagia, H. J. M. Newberg and W. J. Couch 1999 Measurements of $\Omega$ and $\Lambda$ from 42 high-redshift supernovae. Astrophys. J., $\mathbf{5 1 7}$ $565-586$

Pierseaux, Y. 2014 Minkowskian solution of general relativity with cosmological constant and the accelerating universe. J. Mod. Phys., 5: 1725-1732

Richards, F. J. 1959 A flexible growth function for empirical use J. Exp. Bot., 10: 290-300

Riess, A. G., A. V. Filippenko, P. Challis, A. Clocchiatti, A. Diercks, P. M. Garnavich, R. L. Gilliland, C. J. Hogan, S. Jha, R. P. Kirshner, B. Leibundgut, M. M. Phillips, D. Reiss, B. P. Schmidt, R. A. Schommer, R. C. Smith, J. Spyromilio, C. Stubbs, N. B. Suntzeff and J. Tonry 1998 Observational evidence from supernovae for an accelerating universe and a cosmological constant. Astron. J., 116: 1009-1038

Shimojo, M. 2014 An application of Bondi K-factor to the preliminary investigation into some natural phenomena. J. Fac. Agr., Kyushu Univ., 59: 301-303

Shimojo, M. 2015a A size expansion analysis using a toy model based on the equivalence between Bondi K-factor and exponential function. J. Fac. Agr., Kyushu Univ., 60: 93-96

Shimojo, M. 2015b Problems in size expansion analysis based on hypothetical relationships between scale factor, exponential function and Bondi K-factor. J. Fac. Agr., Kyushu Univ., 60 399-403

Shimojo, M. 2016a Mathematical structure common to animal growth analysis and space expansion analysis. J. Fac. Agr. Kyushu Univ., 61: 89-93

Shimojo, M. 2016b A supplement to mathematical structure common to animal growth analysis and space expansion analysis. $J$. Fac. Agr., Kyushu Univ., 61: 287-291

Shimojo, M., Y. Nakano, M. Tobisa and T. Shao 2012 Deriving five growth functions from Bertalanffy function based on symmetry and complexity. J. Fac. Agr., Kyushu Univ., 57: 151-152

Sitnikov, L. S. 2006 Hubble's law and superluminity recession velocities. arXiv.astro-ph/0602102v2

Taniguchi, Y. 2005 The Mystery of the Dark Universe (Blue Backs B1496). Kodansha, Ltd., Tokyo (written in Japanese)

Tatum, E. G., U. V. S. Seshavatharam, S. Lakshminarayana 2015 The basics of flat cosmology. Int. J. Astron. Astrophys., 5 116-124

Tegmark, M. 2014 Our Mathematical Universe. Alfred A. Knopf, Inc., New York.

Tsujikawa, S. 2013 Lecture on Modern Cosmology. Saiensu-sha Co., Ltd. Publishers, Tokyo. (written in Japanese)

Weinberg, S. 2008 Cosmology. Oxford University Press Inc., New York. 